\title{
A comparison of F-region ion velocity observations from the EISCAT Svalbard and VHF radars with irregularity drift velocity measurements from the CUTLASS Finland HF radar
}

\author{
J. A. Davies, T. K. Yeoman, M. Lester, S. E. Milan \\ Radio and Space Plasma Physics Group, Department of Physics and Astronomy, University of Leicester, \\ University Road, Leicester, LE1 7RH, UK \\ e-mail: jaq@ion.le.ac.uk \\ Received: 24 September 1999 / Revised: 21 February 2000 / Accepted: 24 February 2000
}

\begin{abstract}
During August 1998, the UK EISCAT special programme SP-UK-CSUB, which combines operation of both the mainland VHF and Svalbard UHF incoherent scatter radars, was run for several hours around magnetic midnight on four consecutive days. The CUTLASS Finland HF coherent scatter radar was, at these times, operating in a discretionary mode, sounding on all 16 beams, one at high-time resolution. This study presents a comparison of the velocities measured by coherent and incoherent techniques during the SP-UKCSUB experiments. Agreement, particularly between the ion velocities measured by the EISCAT Svalbard radar and irregularity drift measurements by the Finland radar, is remarkable, thereby validating the scientific integrity of both data sets. This work highlights the substantive contribution to our understanding of the solar-terrestrial environment which can be made by use in concert of incoherent and HF coherent scatter radars.
\end{abstract}

Key words: Ionosphere (ionospheric irregularities; plasma convection; instruments and techniques)

\section{Introduction}

Irregularity drift velocity measurements by coherent scatter radars have been compared to bulk plasma velocity measurements by other instrumentation, including satellite borne probes and incoherent scatter radars. Haldoupis and Schlegel (1990), and authors referenced therein, confirmed experimentally previous theoretical evidence that the phase velocity of E-region irregularities, measured with the STARE VHF coherent scatter radar, is limited in magnitude to near the ionacoustic speed. Evidence, both theoretical (e.g. Keskinen

Correspondence to: J. A. Davies and Ossakow, 1983) and observational (Villain et al., 1985; Ruohoniemi et al., 1987; Baker et al., 1990; Davies et al., 1999), suggests that the motion of F-region irregularities measured by HF coherent scatter radars, which, unlike their VHF or UHF counterparts, are not limited to E-region observation at high-latitude due to the refractive nature of radio propagation at HF, is determined by the ambient plasma flow. This has also been found to be true of irregularities generated artificially in the F-region by high-power heating facilities (e.g. Eglitis et al., 1998).

The studies by Villain et al. (1985), Ruohoniemi et al. (1987) and Baker et al. (1990) were based on data taken over a restricted time interval and, consequently, on a very limited number of velocity pairs. In contrast, Davies et al. (1999) compared EISCAT UHF radar observations from over 1000 hours of common programme 1 (CP-1) and common programme 2 (CP-2) experiments to the drift velocity of natural irregularities, the latter measured by the CUTLASS Finland HF radar. Despite their extensive database, Davies et al. (1999) still found themselves limited to a relatively small number of velocity pairs, the EISCAT mainland site being by no means optimally located within the Finland radar field-of-view from which to receive F-region returns. Here, irregularity drift speeds measured by the CUTLASS Finland radar are compared to ion velocity observations by the EISCAT mainland VHF radar and the EISCAT Svalbard radar (ESR).

\section{Experimental arrangement}

The UK EISCAT special programme SP-UK-CSUB, which combines operation of both the VHF radar and the ESR, was run over four four-hour intervals commencing at 21 UT on 20, 21, 22 and 23 August 1998. During these times, the CUTLASS radar at Hankasalmi in Finland (e.g. Davies et al., 1999 and references therein) was operating in a discretionary mode in which all 16 beams (numbered clockwise 0 to 15 ) were sounded at a dwell time of $2 \mathrm{~s}$, but with the radar returning to 
sound on beam 9 between each; the scan is said to camp on beam 9. In total 75 range gates were sampled along each beam with a gate length of $45 \mathrm{~km}$, and a range to the first gate of $180 \mathrm{~km}$.

The ESR, located at Longyearbyen (e.g. Wannberg et al., 1997) was, during SP-UK-CSUB, directed southward, with a geographic azimuth of $161.6^{\circ}$ and an elevation of $31.0^{\circ}$. The ESR beam is aligned, at least in azimuth, along beam 9 of the CUTLASS Finland radar field-of-view. The ESR was transmitting the GUP0 radar code, a multi-frequency long pulse. Received signals were integrated over $10 \mathrm{~s}$ and the data subsequently analysed at a temporal resolution of $60 \mathrm{~s}$. Analysis of ESR data is such that the user can vary the range resolution, in this case with gating of $12 \mathrm{~km}$ below a range of $330 \mathrm{~km}$, which corresponds to an altitude of around $175 \mathrm{~km}$, and $36 \mathrm{~km}$ range gates above $330 \mathrm{~km}$.

In SP-UK-CSUB, the EISCAT VHF radar at Tromsø operated in a split beam mode with one beam (beam 2) directed along the boresight which corresponds to a geographic azimuth of $359.5^{\circ}$ and the other (beam 1) phased $14.5^{\circ}$ west to an azimuth of $345.0^{\circ}$; both beams are at a elevation of $30^{\circ}$. In SP-UK-CSUB, which is identical to the VHF common programme CP-4, long pulse and power profile codes are transmitted. The long pulse, to which data this study is limited, provides lineof-sight observations, on each beam, over 20 gates of range resolution $65.3 \mathrm{~km}$ with the first gate centred at a range of $533.0 \mathrm{~km}$. Like those from Svalbard, VHF observations were analysed at $1 \mathrm{~min}$ temporal resolution. Beam 1 of the VHF radar is aligned in azimuth along beam 7 of the Finland radar whereas beam 2 is less convenient with regard the present study in this respect, crossing as it does beams 8,9 and 10 of the Finland radar.

Figure 1 presents a map on which is depicted the field-of-view of the CUTLASS Finland radar, beams 7 and 9 of which are illustrated explicitly. Moreover, coverage by the ESR is represented by a solid line and the VHF radar beams are indicated by dashed lines. The large filled circles represent the locations of the radars themselves and the cross within a circle, the geographic pole; the magnetic pole is indicated by the small filled circle.

\section{Results}

The four runs of SP-UK-CSUB are not equally useful with regard a comparison of irregularity drift velocity and F-region ion velocity. During the first run, 20 August 2100 UT-21 August 0100 UT, there are a limited number of coherent echoes observed by the Finland radar within the viewing range of the ESR and VHF radars, mainly in the lower CUTLASS range gates of the common field-of-view. The vast majority of these returns are, however, characterised by very narrow spectral widths and low velocities and, as such, have been identified as ground scatter. For the purpose of the present study this interval is not considered further.

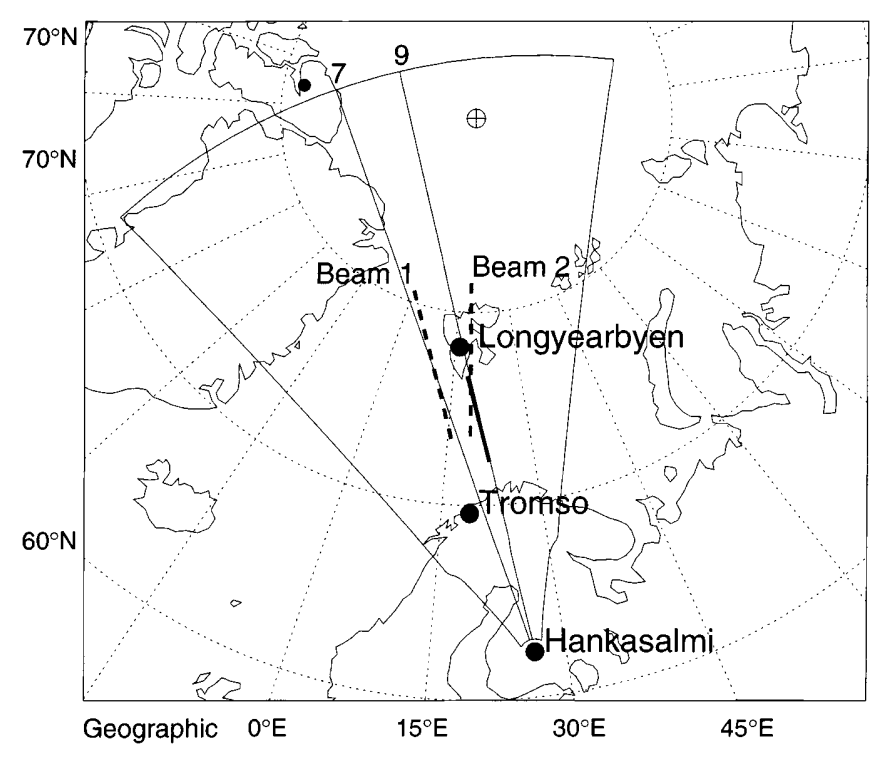

Fig. 1. The geographic location of the field-of-view of the CUTLASS Finland radar, the ESR beam (solid line) and both the VHF radar beams (dashed lines). Beams 7 and 9 of the CUTLASS radar are highlighted explicitly. The locations of the radars are represented by large solid circles. The smaller solid circle indicates the position of the geomagnetic pole while the position of the geographic pole is represented as a cross within a circle

During the third run, ionospheric scatter is observed by the Finland radar within the common viewing area only between 2100 and 2200 UT on August 22. Over the other two runs, there is a preponderance of coherent scatter identified as being of ionospheric origin within that portion of the Finland field-of-view accessible to the incoherent scatter radars.

Figure 2 presents long pulse observations of line-ofsight ion velocity from 2100 UT on 21 August to 0100 UT on 22 August 1998 from the EISCAT Svalbard radar (Fig. 2a), and beams 1 and 2 of the mainland VHF radar (Fig. 2b, c, respectively). Figure 2d, e depicts line-of-sight irregularity drift velocity measurements along beam 7 and beam 9, respectively, of the CUTLASS Finland radar. Coherent returns identified as arising from ground scatter are excluded as indeed are all Finland radar data with a line-of-sight irregularity drift velocity of magnitude less than $50 \mathrm{~m} \mathrm{~s}^{-1}$; the latter is an attempt to remove potentially misidentified ground scatter (e.g. Davies et al., 1999). Velocities are plotted as a function of geomagnetic latitude. Velocities are defined as being positive towards the radar, except in the case of the Svalbard radar where positive velocities represent flow away from the radar in order to retain a consistency between the general orientations of the observations. An in-depth assessment of the geophysical processes arising during this interval is not attempted here, that being the subject of a forthcoming study.

The vagaries of propagation in the HF band is such that the altitude from which scatter originates is not well determined. Elevation angle-of-arrival measurements by the Finland system are consistent with the ionospheric scatter observed in the common viewing area of the three instruments during the SP-UK-CSUB experi- 

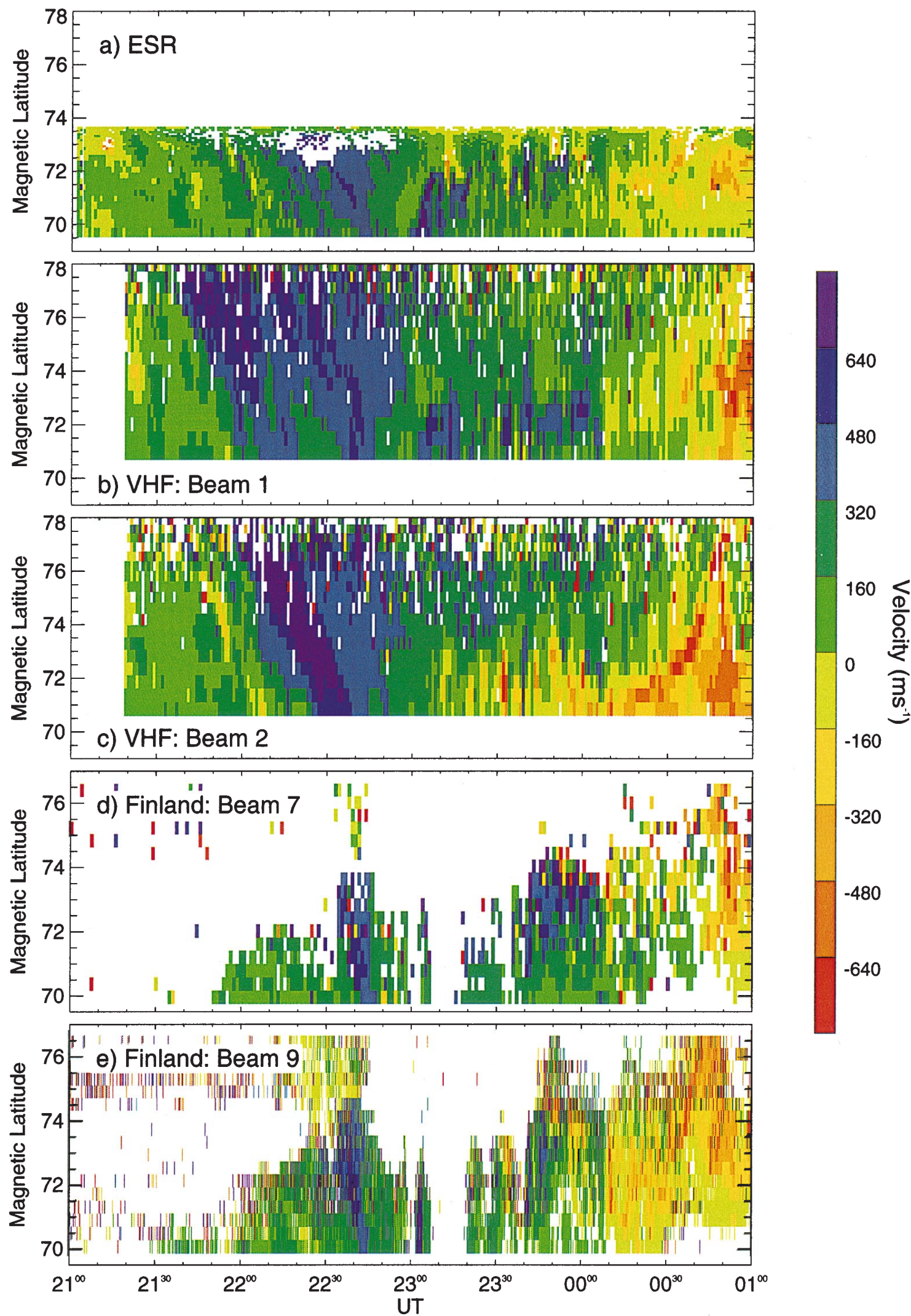

Fig. 2a-e. Line-of-sight velocity measurements during the SP-UKCSUB experiment run from 2100 UT on 21 August to 0100 UT on 22 August, 1998. Successive panels illustrate data from a the EISCAT
Svalbard radar, b beam 1 of the VHF radar, $\mathbf{c}$ beam 2 of the VHF radar, $\mathbf{d}$ beam 7 of the CUTLASS Finland radar and $\mathbf{e}$ beam 9 of the Finland radar, each as a function of geomagnetic latitude 
ments, being of F-region origin. The lowest range gate for VHF observations corresponds to an altitude of some $280 \mathrm{~km}$ while the lowest altitude at which the Svalbard radar observes is $108 \mathrm{~km}$. As such, it should be borne in mind that ion velocities in the first six or so ESR range gates, i.e. at altitudes below some $140 \mathrm{~km}$, where ion-neutral collisions are significant, will not be representative of the component of the $\mathbf{E} \times \mathbf{B}$ velocity along the radar line-of-sight.

Even a cursory glance reveals good general agreement between the observations from the coherent and incoherent systems, and, indeed, between the two incoherent scatter radars themselves. Exact agreement is not anticipated due to disparities between the sampling volumes of the systems (the beam width of the incoherent scatter radars being almost an order of magnitude smaller than that of the HF radar) and the integration periods of the data sets; velocities from both the ESR and VHF radars are integrated over $60 \mathrm{~s}$, whereas beam 9 of the Finland radar is integrated for 2 out of every $4 \mathrm{~s}$ and beam 7 for only $2 \mathrm{~s}$ out of a total scan time of $64 \mathrm{~s}$. Further discrepancies will be introduced due to geometrical factors. Finland velocities can be assumed orthogonal to the local magnetic field, this being the condition necessary for coherent scatter. This need not be so for incoherent scatter, and, indeed, is not so here. It should also be noted that in the calculation of the range from which coherent returns are attributed assumes straight line propagation of HF waves, i.e. refraction effects are neglected and group retardation is ignored. Although deviations from free-space propagation have been found to be small, less than the length of a single range cell (e.g. Ruohoniemi et al., 1987), they are, nevertheless, not insignificant, particularly in studies of this type.

Despite these possible sources of discrepancy, many small-scale flow features observed by the Finland radar appear to be mirrored in the incoherent scatter observations, for example the equatorward velocity burst observed at around 2300 UT both in beam 9 of the CUTLASS field-of-view and by the Svalbard radar. The relationship between F-region ion velocity and irregularity drift velocity is investigated on a more quantitative basis by employing appropriate observations from all but the first SP-UK-CSUB experiment.

Ion velocities measured by the ESR are compared statistically to the irregularity drift velocities from beam 9 of the Finland radar in Fig. 3a while velocities from beam 1 of the VHF radar are compared to drift speeds from Finland Beam 7 in Fig. 3b; the sign convention of the previous figure is maintained, with positive velocities directed towards the Finland radar. As with the previous figure, all Finland radar data with a line-of-sight irregularity drift speed less than $50 \mathrm{~m} \mathrm{~s}^{-1}$, in addition to those returns conventionally identified as arising from ground scatter, are excluded. Moreover, a $3 \mathrm{~dB}$ threshold has been applied to the CUTLASS data and a $2 \%$ signal-to-noise ratio level to the EISCAT VHF observations. In the situation where there are multiple estimates of irregularity drift velocity within a single
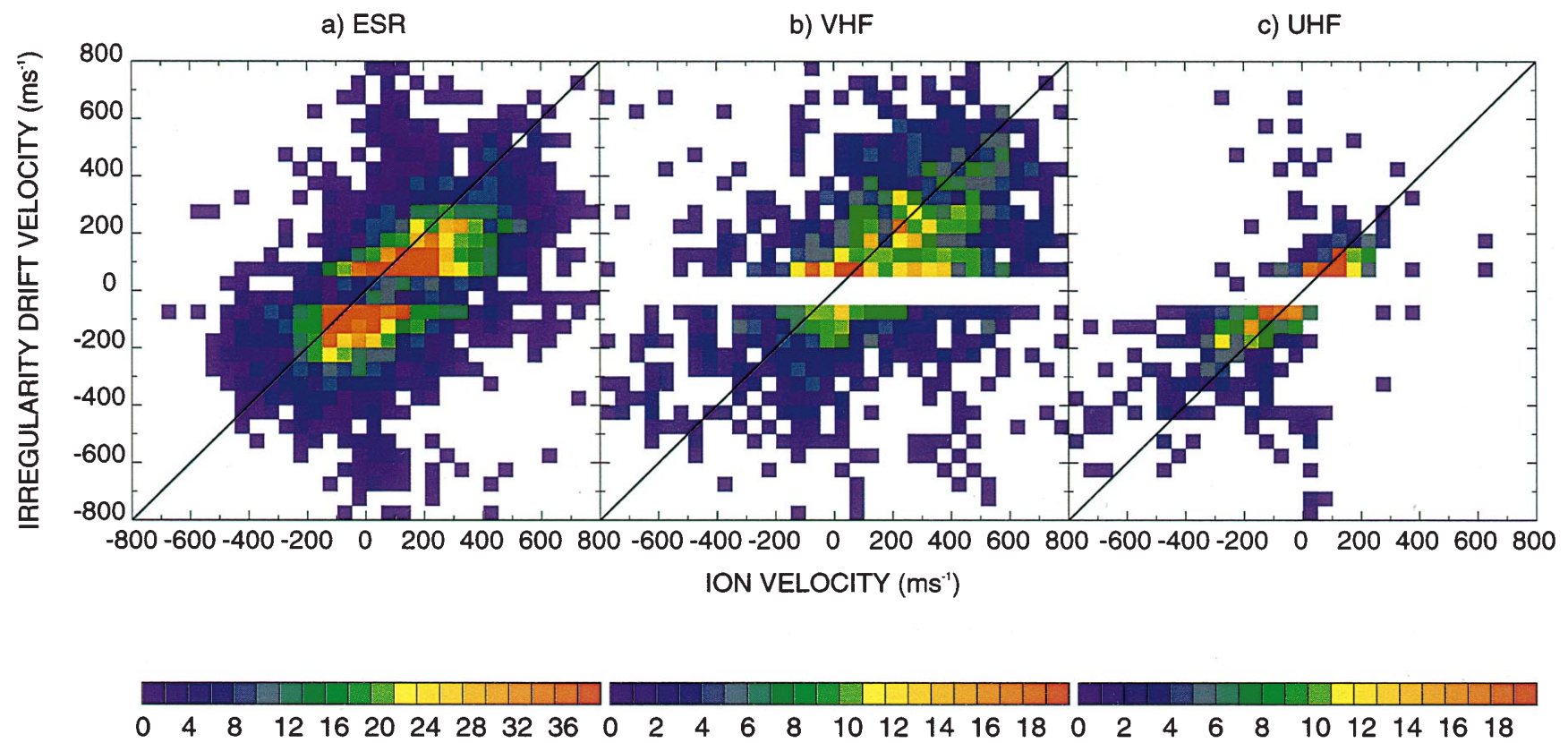

\section{No. OF OCCURRENCES}

Fig. 3a-c. Scattergram of irregularity drift velocity a measured by the Finland radar versus F-region ion velocity measured by the ESR and b along beam 1 of the VHF radar, during the SP-UK-CSUB experiments. Both ion velocity and irregularity drift speed are binned with a width of $50 \mathrm{~m} \mathrm{~s}^{-1}$, with the number of observations in each bin represented by the colour scale to the right. c Reproduces the data from the upper panel of Fig. 5 of Davies et al. (1999), in which ion velocity from the UHF radar is plotted against F-region irregularity drift velocity from the Finland radar, in an identical format to a and b. The solid lines indicate a hypothetical equality of irregularity drift and ion velocity 
EISCAT integration period, these have been averaged together. Moreover, where there are more than one ion velocity observation within a single CUTLASS range gate, as is the case with the ESR, these too have been averaged together. Although coherent returns of velocity less than $50 \mathrm{~m} \mathrm{~s}^{-1}$ have been excluded, the average of the irregularity drift velocity over an EISCAT integration period may still be less than that value. ESR range gates centred below $140 \mathrm{~km}$ altitude are excluded from the analysis, the velocities therein not being representative of magnetised flow.

The line-of-sight ion velocities measured by the ESR and along beam 1 of the VHF radar must be corrected for geometrical effects in order that direct comparison be made with the Finland drift observations. As noted previously, coherent scatter requires the radar wave vector to be near orthogonal to the magnetic field. Although at approximately the same azimuths as beams 7 and 9 of the Finland radar, the ion velocities measured by the incoherent scatter systems are not perpendicular to the magnetic field, rather at an angle determined by the radar beam elevation and direction of the local geomagnetic field. The ion velocities are corrected such that they represent the field-perpendicular flow, albeit assuming that there is no field-parallel motion of the ion population, an assumption the validity of which is discussed in more detail in the subsequent section, and that the radar beam lies in the same plane as the magnetic field, the latter of which is reasonable under these circumstances of a mainly northward directed beam. The solid lines on Fig. 3 indicate a hypothetical equality of irregularity drift and ion velocity for comparative purposes.

\section{Discussion}

These observations, made over a four day interval, provide an exceptional opportunity for the comparison of F-region ion velocity with the drift speed of F-region field-aligned irregularities. This is especially noteworthy given that in the work of Davies et al. (1999) coherent returns from the Finland radar were observed in the vicinity of the EISCAT site for a time totalling less than $4 \mathrm{~h}$ out of an entire database comprising more than $1000 \mathrm{~h}$ of measurements from the two most frequently run UHF common programmes, taken over a three year interval. Due to the geometrical arrangement of the SPUK-CSUB experiment, the present VHF and ESR observations have the advantage over the UHF observations employed in the velocity comparison of Davies et al. (1999) of being simultaneous over a number of CUTLASS Finland range gates. Moreover, the present study concentrates on latitudes poleward of those considered by Davies et al. (1999) where the nature of HF propagation limits Finland returns from the F-region.

Figure $3 c$ reproduces the data from the upper panel of Fig. 5 of Davies et al. (1999), in which ion velocity from the UHF radar is plotted against the drift velocity of F-region irregularities measured by the Finland radar, in an identical format to Fig. 3a, b. In this case the ion velocity plotted is the component of the vector velocity (the UHF being a tristatic system) resolved along the direction of the appropriate Finland beam. Davies et al. (1999) differentiated between E- and F-region drift velocities on the basis of interferometric evidence. Figure 3c, in common with Fig. 3a, b, reveals a number of points corresponding to anomolously high values of irregularity drift velocity, which are attributed to uncorrelated spikes sometimes observed in CUTLASS data (Davies et al., 1999).

The correspondence, not inconsistent with the equivalence of irregularity drift and bulk plasma motion in the F-region, noted by Davies et al. (1999) is surpassed in the present observations, especially with regard the EISCAT Svalbard radar, corrected velocities from which compare remarkably well with the line-of-sight drift velocity measurements from the CUTLASS Finland radar. There is significant spread, however, which may be ascribed to a number of potentially deleterious effects such as discrepant temporal and spatial sampling. There does appear, however, to be a small yet systematic offset, of some 50 to $100 \mathrm{~m} \mathrm{~s}^{-1}$, between the irregularity drift measurements and the ion velocities determined by the Svalbard radar. It is suggested that this is attributable, at least in part, to the assumption that there is no motion of the ion population along the magnetic field direction, used in correcting the incoherent scatter velocity measurements from the Svalbard and VHF radars such that they represent field-perpendicular flows. On the nightside, the general antisunward horizontal motion of the neutral atmosphere, in response to pressure gradients set up by solar heating, will drive ions up the magnetic field with a velocity of some several tens of $\mathrm{m} \mathrm{s}^{-1}$ (e.g. Rodger et al., 1992). Invoking an upward field-aligned velocity of this order does indeed significantly reduce the offset observed between the HF drift and ESR velocity measurements.

Perhaps one of the most noteworthy features of this work is the number of velocity pairs available for comparison, over 3300 for the ESR and nearly 1500 for the VHF radar compared to a mere 525 simultaneous estimates of UHF ion velocity and F-region irregularity drift speed in the study of Davies et al. (1999); this is even more remarkable given that the latter was derived from a data set more than 50 times as long as the combined lengths of the four runs of SP-UK-CSUB. In addition to the superior geometrical arrangement of the SP-UK-CSUB experiment for a study of this type, with regard the location and extended latitudinal range of the common viewing area, this is possibly also attributable to the conditions associated with the more active part of the solar cycle.

\section{Summary and conclusions}

During August 1998, the UK EISCAT special programme SP-UK-CSUB, combining operation of both the mainland VHF and Svalbard incoherent scatter radars, was run in conjunction with discretionary mode 
operation of the CUTLASS Finland HF radar. Line-ofsight velocity measurements from the three instruments exhibit a consistency even with regard to small-scale features in the convective flow pattern. The geometrical arrangement of the experiment permits, moreover, the direct statistical comparison of line-of-sight F-region ion velocities measured by the ESR and along one beam of the VHF radar with irregularity drift velocity measurements from the Finland radar.

Statistical comparison of ion velocities measured by the ESR and irregularity drift measurements by the Finland radar is particularly satisfying, despite a number of potential sources of discrepancy, demonstrating once again that the CUTLASS system provides an invaluable spatial context in which to interpret smallscale features in the high-altitude ionosphere identified by incoherent scatter techniques and, indeed, how significant a contribution to our understanding of solar-terrestrial physics can be made by collaborative use of observations from incoherent and HF coherent scatter radars.

Acknowledgements. The authors wish to acknowledge support from the EISCAT group at the Rutherford Appleton Laboratory and other members of the UK campaign team. Additional thanks are due to N. M. Wade. EISCAT is funded collaboratively by the research councils of Finland, France, the Federal Republic of Germany, Japan, Norway, Sweden and the UK funding body, PPARC. CUTLASS is funded jointly by PPARC, the Swedish Institute for Space Physics and the Finnish Meteorological Institute.

The Editor-in-chief thanks C. Senior for her help in evaluating this paper.

\section{References}

Baker, K. B., R. A. Greenwald, J. M. Ruohoniemi, J. R. Dudeney, M. Pinnock, P. T. Newell, M. E. Greenspan, and C. I. Meng, Simultaneous HF radar and DMSP observations of the cusp, Geophys. Res. Lett., 17, 1869-1872, 1990.

Davies, J. A., M. Lester, S. E. Milan, and T. K. Yeoman, A comparison of velocity measurements from the CUTLASS Finland radar and the EISCAT UHF system, Ann. Geophysicae, 17, 892-902, 1999.

Eglitis, P., T. R. Robinson, M. T. Rietveld, D. M. Wright, and G. E. Bond, The phase speed of artificial field-aligned irregularities observed by CUTLASS during HF modification of the auroral ionosphere, J. Geophys. Res., 103, 2253-2259, 1998.

Haldoupis, C., and K. Schlegel, Direct comparison of $1-\mathrm{m}$ irregularity phase velocities and ion acoustic speeds in the auroral E-region ionosphere, J. Geophys. Res., 95, 18989-19000, 1990.

Keskinen, M. J., and S. L. Ossakow, Theories of high-latitude ionospheric irregularities - A review, Radio Sci., 18, 1077-1091, 1983.

Rodger, A. S., R. J. Moffett, and S. Quegan, The role of ion drift in the formation of ionisation troughs in the mid- and high-latitude ionosphere - A review, J. Atmos. Terr. Phys., 54, 1-30, 1992.

Ruohoniemi, J. M., R. A. Greenwald, K. B. Baker, J.-P. Villain, and M. A. McCready, Drift motions of small-scale irregularities in the high-latitude F-region: an experimental comparison with plasma drift motions, J. Geophys. Res., 92, 4553-4564, 1987.

Villain, J.-P., G. Caudal, and C. Hanuise, A SAFARI-EISCAT comparison between the velocity of F-region small-scale irregularities and the ion drift, J. Geophys. Res., 90, 8433-8443, 1985.

Wannberg G., L. Wolf, L.-G. Vanhainen, K. Koskenniemi, J. Röttger, M. Postila, J. Markkanen, R. Jacobsen, A. Stenberg, R. Larsen, S. Eliassen, S. Heck, and A. Huuskonen, The EISCAT Svalbard radar: A case study in modern incoherent scatter radar system design, Radio Sci., 32, 2283-2307, 1997. 